\title{
Sol-Gel Synthesis and Adsorption Properties of Mesoporous Silica with Mercapto Groups
}

\author{
Svetlana A. Novikova*, \\ Vladimir A. Parfenov and Julia N. Zaitseva \\ Institute of Chemistry and Chemical Technology SB RAS \\ FRC «Krasnoyarsk Science Center SB RAS» \\ 50/24 Akademgorodok, Krasnoyarsk, 660036, Russia
}

Received 12.07.2018, received in revised form 24.10.2018, accepted 30.11.2018

The mercapto-functionalized mesoporous silicas have been synthesized via a surfactant-templated sol-gel route using the Pluronic 123 as a directing agent and investigated by different physicochemical methods. Various amounts of (3-mercaptopropyl)trimethoxysilane (MPTMS) from 10 to $40 \%$ relative to TEOS have been introduced during the sol-gel synthesis with and without TEOS prehydrolysis. The specific surface of the sorbents obtained is 300 to $700 \mathrm{~m}^{2} / \mathrm{g}$. The accessibility of the surface is ensured by the pores with a diameter of 3 to $5 \mathrm{~nm}$, which is confirmed by a directly proportional relationship between the sorption capacity and the sulfur content in the samples obtained. $\mathrm{Hg}(\mathrm{II})$ and Pd(II) sorption capacities are numerically comparable and found to be $0.7 \mathrm{mmol} / \mathrm{g}$ for sorbents containing $10 \%$ MPTMS, and about $1 \mathrm{mmol} / \mathrm{g}$ for sorbents containing $20 \%$ MPTMS.

Keywords: mesoporous mesostructured silica, SiO2, SBA-15, (3-mercaptopropyl)trimethoxysilane, MPTMS, Pluronic 123, sol-gel method, co-condensation, sorption, palladium, mercury.

Citation: Novikova S.A., Parfenov V.A., Zaitseva Ju.N. Sol-gel synthesis and adsorption properties of mesoporous silica with mercapto groups, J. Sib. Fed. Univ. Chem., 2018, 11(4), 518-530. DOI: 10.17516/1998-2836-0096.

(C) Siberian Federal University. All rights reserved

* Corresponding author E-mail address: snovik.chem@gmail.com 


\title{
Золь-гель синтез и адсорбционные свойства мезопористого силиката с меркаптогруппами
}

\author{
С.А. Новикова, В.А. Парфёнов, Ю.Н. Зайцева \\ Институт химии и химической технологии СО РАН \\ ФИЦ «Красноярский научный цุентр СО РАН» \\ Россия, 660036, Красноярск, Академгородок, 50/24
}

\begin{abstract}
Меркапто-функиионализированные мезопористые силикаты были синтезированы золь-гель методом с использованием направляющего агента Pluronic 123 и исследованы различными физико-химическими методами. При синтезе сорбентов вводили различное количество (3-меркаптопропил)триметоксисилана (MPTMS) от 10 до $40 \%$ относительно TEOS с предгидролизом TEOS и без него. Удельная внутренняя поверхность полученных сорбентов составляет от 300 до $700 \mathrm{M}^{2} / 2$. Доступность поверхности обеспечивается порами диаметром от 3 до 5 нм, что подтверждается прямо пропорииональной связью сорбционной емкости и содержания серы в образцах. Сорбционная емкость по Hg(II) и Pd(II) численно сопоставима и составляет до 0,7 ммоль/ для сорбентов, содержащих $10 \%$ MPTMS, и около 1 ммоль/г - для сорбентов, содержащих $20 \%$ MPTMS.
\end{abstract}

Ключевые слова: мезопористый мезоструктурированный материал, $\mathrm{SiO}_{2}, \quad \mathrm{SBA}-15$, (3-меркаптопропил)триметоксисилан, MPTMS, Плуроник 123, диоксид кремния, золь-гель синтез, соконденсация, сорбция, палладий, ртуть.

\section{Введение}

Мезопористые мезоструктурированные материалы (ММM) - класс веществ, характеризующийся надмолекулярным дизайном периодически повторяющихся структурных деталей в нанометровом диапазоне. Указанные материалы имеют высокую площадь внутренней поверхности, большие по объему и одинаковые по размеру поры. Это следующий за цеолитами тип каркасных материалов, синтез которых основан на молекулярном темплатировании. В отличие от цеолитов, где в качестве темплата выступают отдельные молекулы или ионы, темплатирование в МММ происходит на молекулярных жидкокристаллических поверхностях, собранных из лиофильных молекулярных частиц поверхностно-активных веществ. Исследованный диапазон варьирования диаметра пор, исходя из литературных данных, составляет 2-30 нм. Наибольшее внимание исследователей получили силикатные материалы MCM-41 и SBA-15 с условным составом кремнезема $\mathrm{SiO}_{2}$ [1-10]. Сотоподобный каркас силикатной структуры образует стабильную подложку для различных молекулярных процессов, что открывает широкие возможности применения в катализе, адсорбции, молекулярной сепарации, оптике, электронике, нанокастинге и в других областях. Модификация поверхности органическими и неорганическими частицами позволяет настраивать поверхностные свойства и регулировать взаимодействие «хозяин-гость», изменяя, например, гидрофиль- 
ные и гидрофобные свойства, конструкционные и сорбционные характеристики, коэффициент преломления, диэлектрическую проницаемость и другие свойства [1-18]. С точки зрения структуры функционализация может быть ориентирована на внутреннюю поверхность стенки и пространство пор. Большое количество работ выполнено по функционализации поверхности кремнийорганическими препаратами с функциональными группами, такими как амино- и меркаптогруппы. Описано две разновидности синтеза: одностадийная, в которой функционализатор вводится на стадии «сборки» силикатного каркаса материала, и двухстадийная, предполагающая постсинтетическую реакцию прививки на силикатную поверхность органосиликатного молекулярного фрагмента [19-26]. В литературе представлены многочисленные данные по функционализации с целью создания сорбентов для извлечения металлов из растворов $[5,9,11,19-26]$. В качестве наиболее впечатляющего, но по-прежнему не повторенного результата можно упомянуть работу Лю с соавторами [20], получившими серосодержащий сорбент с сорбционной емкостью (CE) по $\mathrm{Hg}^{2+}$ примерно 3 ммоль/г. По замечанию некоторых авторов, воспроизводимость упомянутых синтезов достаточно низка. Проблемой является блокировка пор органосиликатными полимерными фрагментами, а также случайное распределение «прививок» по поверхности, что может лимитировать дальнейшее применение [27-31].

В основу одного из направлений совершенствования функционализированных силикатных матриц положена идея покрытия внутренней поверхности силиката дополнительным слоем полимерной органической матрицы. Последняя, по существу, играла роль органической смолы, к которой привиты функциональные группы. Предполагалось, что органонеорганический композит не будет проявлять такие отрицательные качества органических смол, как набухание, и одновременно сохранит их высокую емкость. Периодические мезопористые органосиликаты являются представителями такого класса материалов. Для их синтеза наряду с тетраэтоксисиланом (TEOS) в качестве источника кремнезема используются силсесквиоксаны, например бис-триэтоксисилилэтан [32, 33]. Было показано, что получаемые функционализированные продукты обладают высокой емкостью по различным ионам металлов. Однако такие синтезы значительно удорожают сорбент [34, 35].

В литературе также описан синтез органосиликатного композита из реакционной среды, содержащей три основных компонента: источник кремнезема (TEOS), направляющий агент (блок-сополимер) и растворимая смола $[39,40]$. Продукт имеет однородный пористый каркас, состоящий из органической и неорганической компоненты. Каналы между пор остаются незанятыми. Работоспособность материала была продемонстрирована в качестве подложки для высокоактивных палладиевых и титановых катализаторов.

В данной работе рассматривается одностадийный золь-гель синтез мезопористых мезоструктурированных сорбентов из трехкомпонентных синтетических сред, в которых в качестве источника кремния и функционализатора выступают TEOS и MPTMS соответственно. В качестве директирующего агента выбран Pluronic 123. Таким образом, получаемые материалы являются аналогами SBA-15. Эти гибридные органонеорганические материалы находят множество приложений в сорбции и катализе. Среди потенциальных приложений большой интерес представляет процесс выделения тяжелых металлов и МПГ из различных промышленных растворов с использованием селективных сорбентов с функционализированной поверхностью.

$$
-520-
$$




\section{Экспериментальная часть}

\section{Используемые реактивы}

Тетраэтоксисилан (TEOS): $\mathrm{Si}\left(\mathrm{C}_{2} \mathrm{H}_{5} \mathrm{O}\right)_{4}$, ч.д.а., ТУ 6-09-3687-74; этанол-ректификат (EtOH),

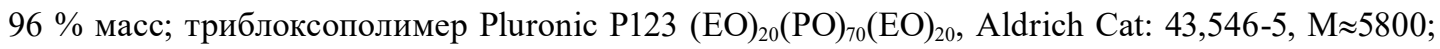
3-меркаптопропилтриметоксисилан (MPTMS): $\mathrm{C}_{6} \mathrm{H}_{16} \mathrm{O}_{3} \mathrm{SSi}$ (Aldrich, Cat.: 175617); хлороводородная кислота: $\mathrm{HCl}$, ч.д.а; растворы ртути(II), палладия(II).

Золь-гель синтез

В экспериментах количество использованного органического силана (MPTMS) варьировали по молярному соотношению: $\mathrm{x}=\mathrm{MPTMS} /(\mathrm{MPTMS}+\mathrm{TEOS})$, равному 10, 20, 40 \%. Для обозначения синтезированных в данном разделе образцов применялось обозначение $\mathrm{Mn}$, где M - меркаптогруппа, $\mathrm{n}$ - номер образца. Синтез образцов с $\mathrm{n}=1,3,5$ был выполнен с предварительным гидролизом TEOS в соответствии с [41, 42]. Образцы с $\mathrm{n}=2,4$ синтезированы без предгидролиза TEOS [43].

4 г Р123 растворяли при комнатной температуре в 125 г $\mathrm{HCl}$ 1,9 М. После нагревания до $40{ }^{\circ} \mathrm{C}$ добавляли 0,037 моль (7,7 г) TEOS и выдерживали при перемешивании в течение 45 мин до добавления 3-меркаптопропилтриметоксисилана (MPTMS). Пауза в 45 мин является оптимальным временем для предварительного гидролиза TEOS, по данным [41]. Количество добавляемого MPTMS составляло $10 \%$ (M1, M2) и $20 \%$ (M3, M4) и $40 \%$ (M5) относительно TEOS. Реакционную смесь перемешивали в течение 20 ч при $40{ }^{\circ} \mathrm{C}$. Далее в том же сосуде проводилась гидротермальная обработка (ГТО) в течение 48 ч при $80^{\circ} \mathrm{C}$ в статических условиях. Затем продукт фильтровали и высушивали на воздухе. Образцы M2 и M4 были получены без предгидролиза TEOS, т.е. TEOS и MPTMS смешивали и вводили одновременно в реакционную смесь.

Процесс конденсации при синтезе образцов M1 и M2 (10 \% MPTMS) визуально проходил без образования геля. При синтезе образцов М3 и M4 (20 \% MPTMS) по истечении 10 мин после добавления силикатсодержащих компонентов, особенно в виде смеси (TEOS+MPTMS), образовался гель, который впоследствии (еще через 30 мин) перешел в порошковую взвесь. Образец M5 (40 \% MPTMS) сразу после добавления MPTMS превратился в густой, трудно перемешиваемый клееподобный гель - коллоид, который приобрел порошкообразную консистенцию только после стадии ГТО. В целом следует отметить, что соотношение ПАB:TEOS, превышающее значение 1:60, всегда приводит к образованию геля. Некоторые детали проведенных синтезов представлены в табл. 1.

Поскольку в состав органической части органосиликатного композита входили меркаптопропильные фрагменты, удаление органической части путем окислительного отжига было невозможно. Для удаления ПАВ использовали экстракционный метод.

Известно, что проксанолы (плуроники) состава $\mathrm{HO}\left(\mathrm{C}_{2} \mathrm{H}_{4} \mathrm{O}\right)_{m}\left(\mathrm{C}_{3} \mathrm{H}_{6} \mathrm{O}\right)_{\mathrm{n}}\left(\mathrm{C}_{2} \mathrm{H}_{4} \mathrm{O}\right)_{m}$ при $\mathrm{n}>\mathrm{m}+\mathrm{m}$ ' растворимы в неполярных растворителях. В связи с этим была проведена экстракция $\mathrm{HO}\left(\mathrm{C}_{2} \mathrm{H}_{4} \mathrm{O}\right)_{20}\left(\mathrm{C}_{3} \mathrm{H}_{6} \mathrm{O}\right)_{70}\left(\mathrm{C}_{2} \mathrm{H}_{4} \mathrm{O}\right)_{20}$ неполярным растворителем гексаном. Однако методика экстрагирования ПАВ из образца с помощью гексана не дала требуемого результата. Уменьшение органической части составило всего около $15 \%$ масс. В дальнейшем удаление ПАВ из всех образцов проводили кипячением в спирте с обратным холодильником в течение 24 ч. Соот- 
Таблица 1. Некоторые характеристики продуктов золь-гель синтеза

Table 1. Some characteristics of sol-gel synthesis products

\begin{tabular}{|c|c|c|c|c|}
\hline Образец & $\begin{array}{c}\text { Количество MPTMS } \\
\text { относительно TEOS, } \%\end{array}$ & $\begin{array}{c}\text { Предгидролиз } \\
\text { TEOS }\end{array}$ & $\begin{array}{c}\text { Потеря массы } \\
\text { при отжиге, \% }\end{array}$ & $\begin{array}{c}\text { Потеря массы } \\
\text { при экстракции, \% }\end{array}$ \\
\hline M1 & 10 & + & 55 & 38 \\
\hline M2 & 10 & - & 57 & 49 \\
\hline M3 & 20 & + & 53 & 45 \\
\hline M4 & 20 & - & 54 & 29 \\
\hline M5 & 40 & + & 59 & 48 \\
\hline
\end{tabular}

ношение т:ж = 1:100. Экстрагированный продукт фильтровали и сушили на воздухе. После экстракции к наименованиям образцов было добавлено окончание «Е» (например, M1Е). Небольшая часть образца подвергалась окислительному отжигу при $550{ }^{\circ} \mathrm{C}$ в течение 3 ч для удаления всей органической составляющей с целью изучения состояния силикатной матрицы различными физико-химическими методами. В конце наименований таких образцов добавляли «С» (например, М1C).

\section{Методы характеризации образиов}

Съемку рентгенограмм проводили при использовании $\mathrm{Cu}$ K $\alpha$-излучения на дифрактометре X'Pert PRO с детектором PIXcel (PANalytical), снабженным графитовым монохроматором. Дифрактограммы регистрировались в угловом интервале $2 \Theta 0,40-7,04^{\circ}$ с шагом $0,026^{\circ}$ и временем накопления 120 с в каждой точке.

Адсорбционные измерения поверхности выполняли на приборе ASAP 2420 (Micromeritics) при $\mathrm{T}=77,3$ К в интервале относительных давлений (P/P $)$ 0,06-0,99 с шагом 0,015. Удельную площадь поверхности рассчитывали по модели $B E T$ в интервале (p/p $\mathrm{p}_{\text {o }}$ 0,06-0,25, поверхность микропор вычисляли методом «t-plot», внутренний объем - по методу «Single Point», распределение пор по размерам - по уравнению изотермы $B J H$.

Для оценки количества и типа закрепленных органических функциональных групп мезопористые материалы также анализировали ИК-спектроскопическим методом, проводимым на ИК-спектрометре «Tensor 27» (Bruker, Германия) в интервале 400-4000 см-1. Таблетки для измерений готовили с использованием высушенного $\mathrm{KBr}$. Массовое соотношение образец: $\mathrm{KBr}=1: 400$. Навеска образца составляла около 1,5 мг.

Анализ морфологической структуры образцов проводили методом сканирующей электронной микроскопии на электронных микроскопах Hitachi TM-1000 (ускоряющее напряжение 15 кВ) и НТ7700. Образцы сканировали с различным увеличением. Изображения сканированных объектов сохраняли в виде стандартных JPEG-файлов.

\section{Изучение сорбиионных свойств}

Сорбцию ртути/палладия (II) из хлоридного раствора проводили в статическом режиме по следующей методике (в некоторых случаях с добавкой КCl). Навеску сорбента 50 мг помещали в колбу, приливали 10 мл исследуемого раствора металла (ртути или палладия). Концентрация 
$\mathrm{Pd}(\mathrm{II})$ составляла 3 ммоль/л (1 M HCl), $\mathrm{Hg}(\mathrm{II})-8$ ммоль/л (pH 1,7). Время контакта раствора и сорбента равнялось 24 ч при постоянном перемешивании со скоростью 180 об/мин. После отделения сорбента от раствора фильтрованием (фильтр «синяя лента») в образце определяли содержание элементов (серы, ртути/палладия, хлора, кремния) с применением рентгенофлуоресцентного анализа. Определение элементов проводили на рентгено-флуоресцентном спектрометре Axios Advanced (PANalytical). Таблетки (диаметром 32 мм) для измерения готовили прессованием образца с борной кислотой в качестве связующего.

\section{Результаты и обсуждение}

В табл. 2 представлены структурные и текстурные характеристики образцов. Структурные характеристики изучали методом рентгеновской дифракции. Наличие явных максимумов в малоугловой области на дифрактограммах образцов указывает на образование мезоструктуры. К сожалению, точного отнесения к какому-либо типу структуры выполнить невозможно из-за ограниченного количества пиков. Положение первого пика во всех образцах при экстрагировании смещается в сторону малых углов относительно прокаленных, что указывает на увеличение параметра решетки и рост диаметра пор. Межплоскостное расстояние для основного пика рентгенограммы для прокаленных образцов варьируется в интервале от 79,8 (М4С) до 90,5 $\AA$ (М2С), для экстрагированных - от 81,1 (М4Е) до 102,2 А̊ (М2Е) соответственно. Для образца М5Е первый пик и в целом мезоструктура отсутствуют.

Для изучения поверхностных свойств (текстурных характеристик) образцов использовали метод газовой адсорбции. На рис. 1 приведены изотермы сорбции и графики распределения
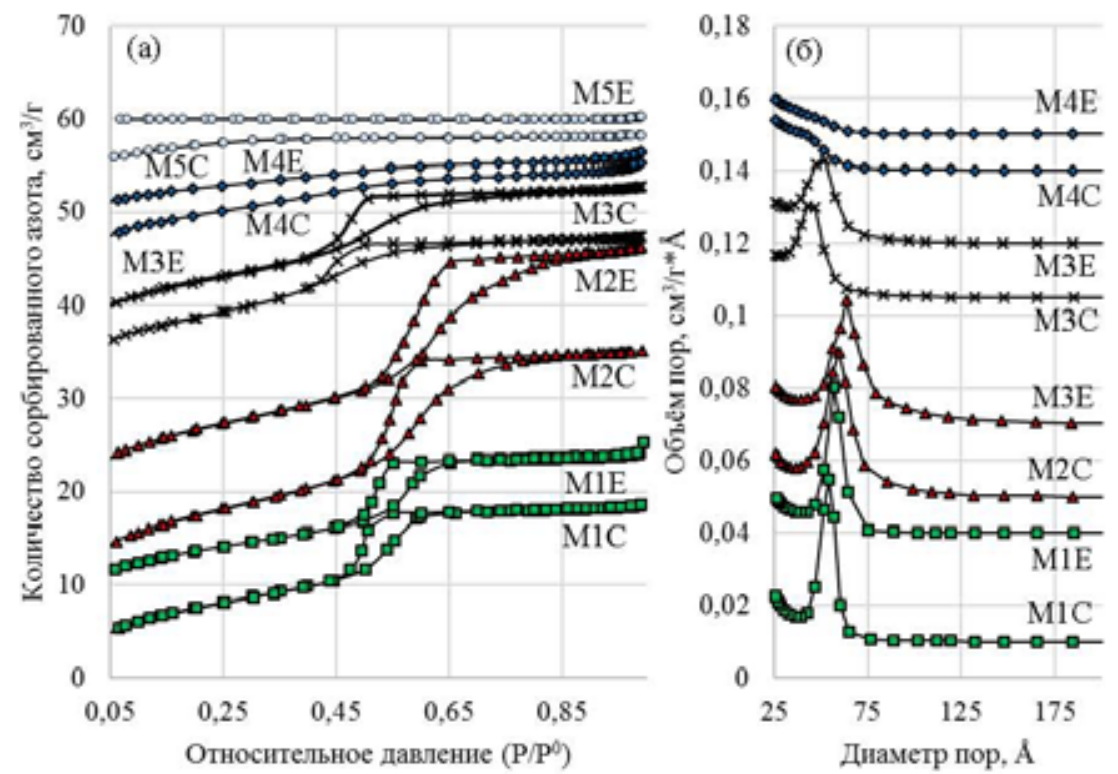

Рис. 1. Изотермы адсорбции азота (а) и распределение пор по размерам (б) для адсорбционной области изотермы. Для образцов М5Е и М5С распределение пор по размерам отсутствует

Fig. 1. Nitrogen adsorption isotherms (a) and pore size distribution (б) for the adsorption region of the isotherm. There is no pore size distribution for the samples M5E and M5C 
Таблица 2. Структурные и текстурные характеристики образцов

Table 2. Structural and textural characteristics of the samples

\begin{tabular}{|c|c|c|c|c|c|c|c|c|}
\hline \multirow[b]{2}{*}{ № } & \multirow[b]{2}{*}{ Образец } & \multicolumn{2}{|c|}{ Структурные характеристики } & \multicolumn{5}{|c|}{ Текстурные характеристики } \\
\hline & & $\begin{array}{c}\text { Положение } \\
\text { 1-го пика, } 2 \Theta \\
\end{array}$ & $\begin{array}{c}\text { Межплоскостное } \\
\text { расстояние d, } \AA\end{array}$ & $\mathrm{S}_{\text {уд }}, \mathrm{M}^{2} / \Gamma$ & $\begin{array}{c}\mathrm{S}_{\text {micro }} \\
\text { (t-plot), } \mathrm{M}^{2} / \Gamma\end{array}$ & $\begin{array}{c}\mathrm{V}_{\mathrm{p}}(\mathrm{BJH}) \\
\mathrm{cm}^{3} / \Gamma\end{array}$ & $\begin{array}{l}\mathrm{V}_{\text {micro }} \\
\mathrm{cm}^{3} / \Gamma\end{array}$ & $\mathrm{D}_{\text {пор }}, \AA$ \\
\hline \multirow{2}{*}{1} & $\mathrm{M} 1 \mathrm{C}$ & 1,46 & 60,5 & 549 & 153 & 0,28 & 0,07 & 20,5 \\
\hline & M1E & - & - & 1,6 & 6 & 0,00 & 0,00 & - \\
\hline \multirow{2}{*}{2} & M2C & 1,13 & 77,9 & 905 & 171 & 0,77 & 0,08 & 34,1 \\
\hline & M2E & 1,09 & 81,0 & 552 & - & 0,58 & - & 42,3 \\
\hline \multirow{2}{*}{3} & M3C & 1,13 & 78,4 & 989 & 95 & 1,04 & 0,04 & 41,9 \\
\hline & M3E & 1,00 & 88,5 & 722 & - & 0,97 & - & 53,6 \\
\hline \multirow{2}{*}{4} & M4C & 1,26 & 69,9 & 849 & 140 & 0,67 & 0,06 & 31,4 \\
\hline & M4E & 1,08 & 81,6 & 623 & - & 0,61 & - & 38,9 \\
\hline \multirow{2}{*}{5} & M5C & 1,28 & 68,7 & 752 & 173 & 0,51 & 0,08 & 26,9 \\
\hline & M5E & 1,26 & 69,9 & 373 & - & 0,27 & - & 29,2 \\
\hline
\end{tabular}

пор по размерам для адсорбционной области изотермы. Адсорбционное измерение удельной поверхности показывает, что чем больше содержание серы в образце, тем ниже удельная поверхность и объем пор (табл. 2). Исключение составляет образец М4Е, обладающий наименее выразительной мезоструктурой (величина S-образного скачка объема на изотерме) по сравнению, например, с М3Е. Далее будет показано, что качество мезоструктуры коррелирует с сорбционной способностью по отношению к металлам. Предельным случаем является образец M5E с содержанием MPTMS 40 \% после экстракции ПАВ, который совсем не имеет скачка на изотерме и практически не обладает свободной внутренней поверхностью (1,6 м²/г). Наличие микропор в силикатах характерно в основном для прокаленных образцов и варьируется в пределах 95-173 м²/г.

На рис. 2 для наглядности приведены ИК-спектры образцов, содержащих 20 и 40 \% функционализатора в сравнении с чистой матрицей SBA-15. Образцы имеют интенсивно выраженные пики на ИК-спектрах в области 2930-2960 см ${ }^{-1}$, отвечающей С-Н-колебаниям, 2550-2600 см-1 колебаниям S-H, 1400-1500 cм-1 - C-C колебаниям, 780 cм$^{-1}$ C-S. Чем выше содержание функционализатора, тем более выражены указанные области.

Наряду с газовой адсорбцией для изучения микроструктуры (морфологии) образцов использовали электронную микроскопию. Материал М5Е с содержанием 40 \% функционализатора представляет собой монолитную пористую структуру (рис. $3 a$ ). По-видимому, это есть следствие высокой концентрации меркаптопропильных групп на внешней поверхности частиц материала, что вызывает взаимную адгезию (слипание) частиц и образование на поверхности агломератов единого, «мягкого» приповерхностного слоя, создающего эффект монолитности. Образцы M1E, M2E, M3E и M4E с содержанием функционализатора от 10 до $20 \%$ имеют упорядоченную гексагональную мезопористую поверхность. На рис. $3 б$ представлен снимок просвечивающей электронной микроскопии на примере образца М3Е. 


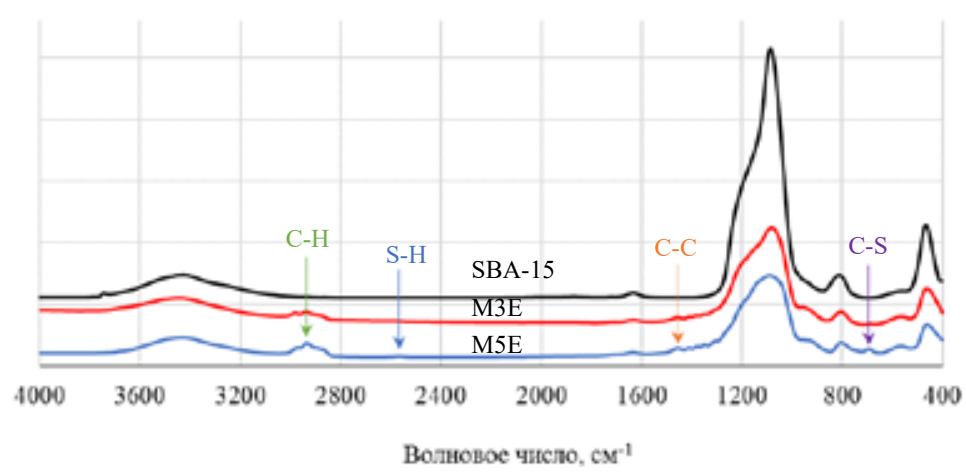

Рис. 2. ИК-спектры некоторых образцов в сравнении с силикатной матрицей SBA-15

Fig. 2. IR-spectra of some samples in comparison with the SBA-15 silicate matrix
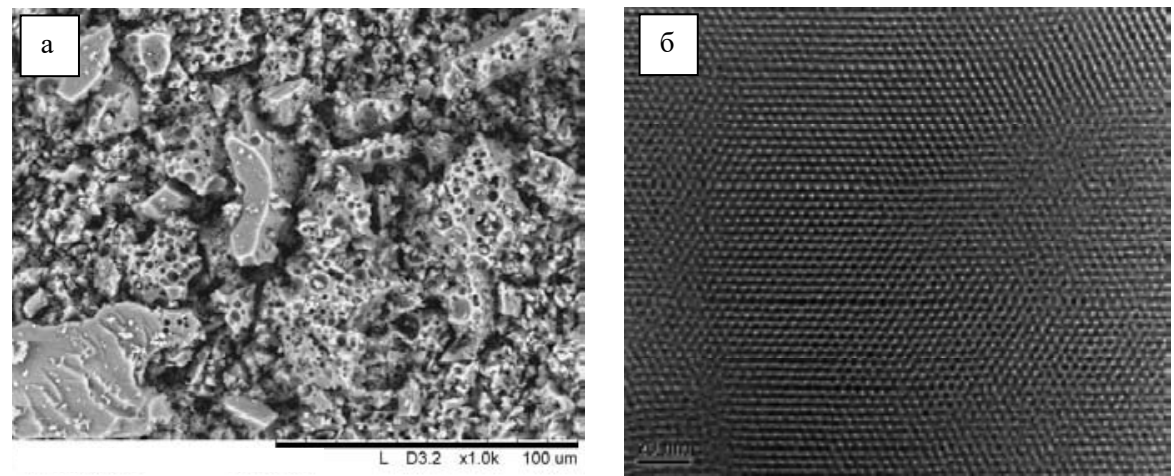

Рис. 3. СЭМ и ПЭМ снимки образцов: a - М5E; б - М3Е

Fig. 3. SEM and TEM images for the samples: a - M5E; $6-\mathrm{M} 3 \mathrm{E}$

Определение предельной емкости сорбентов при извлечении тяжелых

и благородных металлов в различных условиях

Сорбцию палладия (II) проводили из растворов двух типов: $1 \mathrm{M}$ солянокислого раствора $\mathrm{H}_{2} \mathrm{PdCl}_{4}$ и раствора $\mathrm{H}_{2} \mathrm{PdCl}_{4}$ в $\mathrm{KCl}$, при $\mathrm{pH}$ 6,5. Палладий находился в составе аниона $\left[\mathrm{PdCl}_{4}\right]^{2-}$. На рис. 4 приведены результаты сорбции тяжелых и благородных металлов за сутки в статических условиях в виде графической зависимости содержания элементов $(\mathrm{Hg} / \mathrm{Pd}, \mathrm{S}$, $\mathrm{Cl}$ ) в исследуемых образцах. Сорбционная емкость по ртути (II) во всех образцах немного выше емкости по палладию (II). Образец М5E, полученный при 40 \% доли MPTMS относительно TEOS, имеет наивысшую емкость по ртути (до 1,8 ммоль $(\mathrm{Hg}) / \Gamma$ ) при соотношении $\mathrm{Hg} / \mathrm{S}=0,5 \div 0,6$. Однако этот образец практически не сорбирует палладий (меньше 0,1 ммоль/г). Отношение $\mathrm{Hg} / \mathrm{Cl}=0,9$. Возможно, причиной низкой сорбции палладия является специфическое состояние поверхности.

Образцы M3E, M4E (доля MPTMS при синтезе 20 \%) сорбируют палладий значительно эффективнее: до 0,3 ммоль/г из раствора $\mathrm{K}_{2} \mathrm{PdCl}_{4}, \mathrm{Pd} / \mathrm{S}=0,5$ и до 1 ммоль/г из раствора $\mathrm{H}_{2} \mathrm{PdCl}_{4}$, $\mathrm{Pd} / \mathrm{S}=0,4)$. Сорбционная емкость по ртути находится на уровне $0,9-1$ ммоль $/ \Gamma(\mathrm{Hg} / \mathrm{S}=0,5)$. Отношение $\mathrm{Hg} / \mathrm{Cl}=1 \div 2, \mathrm{Pd} / \mathrm{Cl}=0,5 \div 1$. Образцы M1E, M2E (доля MPTMS $10 \%$ ), в одинаковой мере 

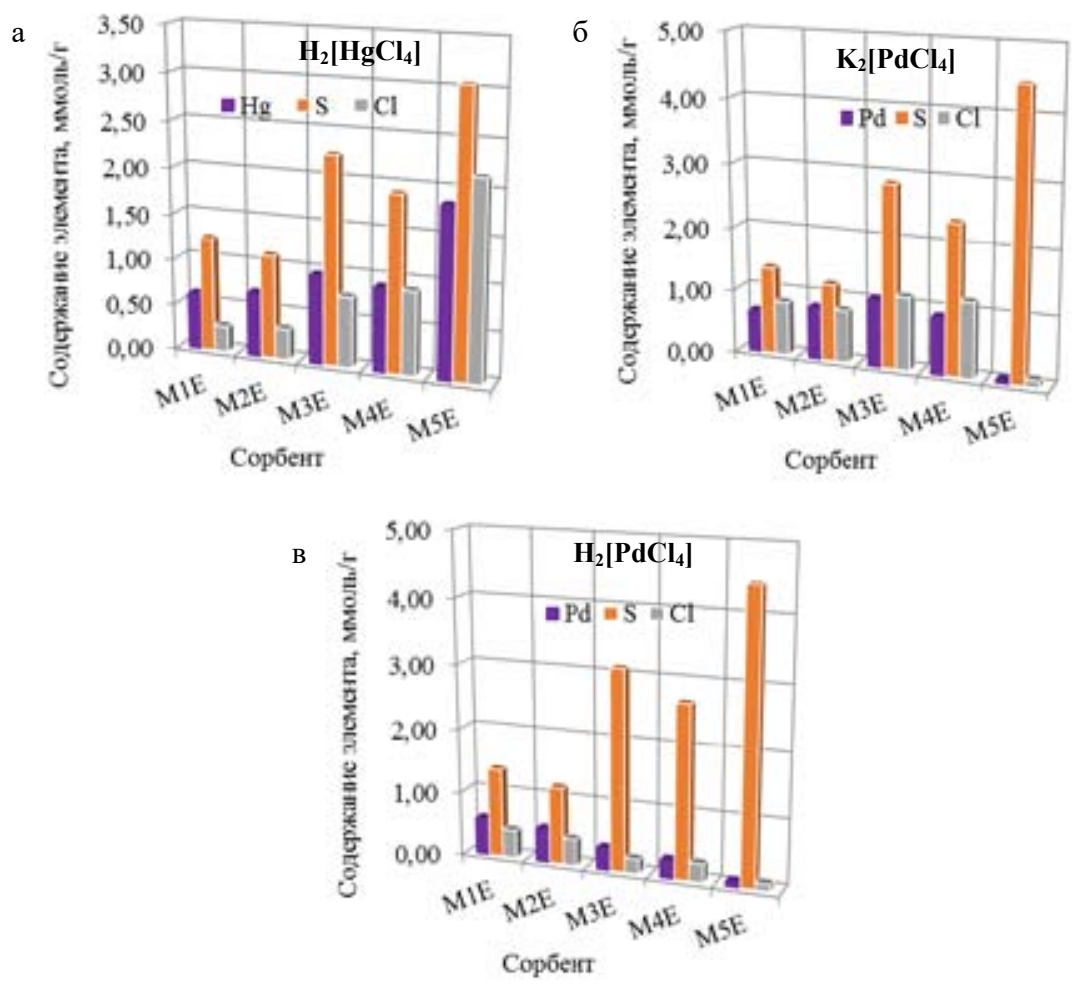

Рис. 4. Относительное содержание элементов $(\mathrm{Hg} / \mathrm{Pd}, \mathrm{S}, \mathrm{Cl})$ для различных образцов: а - в сорбции ртути (II) и палладия (II) из растворов $6-\mathrm{H}_{2} \mathrm{PdCl}_{4}$ и в $-\mathrm{K}_{2} \mathrm{PdCl}_{4}$

Fig. 4. The relative content of elements $(\mathrm{Hg} / \mathrm{Pd}, \mathrm{S}, \mathrm{Cl})$ in different samples: a - in the mercury (II) sorption and palladium (II) sorption from $\sigma-\mathrm{H}_{2} \mathrm{PdCl}_{4}$ and $\mathrm{B}-\mathrm{K}_{2} \mathrm{PdCl}_{4}$ solutions

извлекают и ртуть, и палладий с соотношением $\mathrm{Me} / \mathrm{S}$ в интервале $0,4-0,6$. Максимальная $\mathrm{CE}$ по ртути составляет 0,69 ммоль/г, по палладию - 0,81 ммоль/г. Отношение $\mathrm{Hg} / \mathrm{Cl}=2, \mathrm{Pd} / \mathrm{Cl}=1$.

При рассмотрении графических представлений (рис. 4) следует иметь в виду возникающий вычислительный эффект в методе РФС. Поскольку результаты приведены в относительных весовых процентах, то присутствие «тяжелой» ртути значительно изменяет весовые доли, приходящиеся на другие элементы, и, в частности, занижает содержание серы в образце. Так, в образцах после сорбции ртути содержание серы заметно ниже, чем в образцах, сорбировавших палладий, например 3,1 ммоль/г S (в сорбции $\mathrm{Hg}$ ) против 4,5 ммоль/г S (в сорбции Рd) в рамках одного образца М5Е.

Максимальная сорбционная емкость по палладию для образца МЗЕ составляет 1 ммоль/г. Исходя из данных РФС, на 1 атом серы приходится также 5 «молекул» оксида кремния (т.е. $20 \%$ серы), на 2 атома привитой серы - 1 атом Pd, на 1 атом Pd - 1 атом хлора. За время контакта 5 мин достигается 95 \% емкости от достигнутой за 24 ч. В экспериментах с М4Е максимально достигнутая емкость по палладию (II) в образце составляет 0,9 ммоль/г.

\section{Заключение}

Проведенные исследования показали, что функционализированные мезоструктурированные силикатные сорбенты с высокой сорбционной способностью могут быть успешно получе- 
ны золь-гель методом в присутствии структурно-направляющего агента Pluronic 123. Удельная внутренняя поверхность этих образцов варьируется от 300 до $700 \mathrm{~m}^{2} /$ г. Доступность поверхности обеспечивается порами диаметром от 3 до 5 нм и объемом пор от 0,3 до $1 \mathrm{~cm}^{3} /$ г. Преимуществом золь-гель синтеза модифицированных мезоструктурированных материалов перед методом постсинтенической прививки является одностадийность процесса и высокая степень гомогенизации исходных компонентов.

Однако извлечение ПАВ в случае золь-гель синтеза требует более трудоемкой и дорогой операции - экстрагирования. В результате золь-гель синтеза количество внедренных органических групп соответствует внесенному при синтезе количеству, а не колеблется от образца к образцу от случайных изменений условий синтеза. В этой связи золь-гель синтез более экономичен, так как используется небольшое количество модификатора по сравнению с методом функционализации, где избыток его крайне необходим.

Исследование сорбционных свойств образцов по отношению $\mathrm{Hg}(\mathrm{II})$ и $\mathrm{Pd}(\mathrm{II})$ обнаружило прямо пропорциональную связь сорбционной емкости и содержания серы в образцах, что свидетельствует о доступности поверхности сорбентов. Координация на поверхности отвечает соотношению (1-2)S:1Mе в зависимости от количества используемого функционализатора. Таким образом, две органических группы задействованы на сорбцию одного атома металла. Максимальная сорбционная емкость по ртути, установленная в данном исследовании, составила 1,8 ммоль/г при содержании серы $40 \%$ относительно $\mathrm{SiO}_{2}$, по палладию - 1 ммоль/г при содержании серы $20 \%$ относительно $\mathrm{SiO}_{2}$.

\section{Список литературы}

1. Kresge C.T., Leonowicz M.E., Roth W.J. et al. Ordered mesoporous molecular sieves synthesized by a liquid-crystal template mechanism. Nature 1992. Vol. 359, P. 710-712.

2. Yanagisawa T., Shimizu T., KurodaK. andKato C. Thepreparation ofalkyltrimethylammoniumkaneinite complexes and their conversion to microporous materials. Bull. Chem. Soc. Jpn. 1990. Vol. 63, P. 988-992.

3. Zhao D.Y., Feng J. L., Huo Q. S. et al. Triblock Copolymer Syntheses of Mesoporous Silica with Periodic 50 to 300 Angstrom Pores. Science 1998. Vol. 279, P. 548-552.

4. Corma A. From microporous to mesoporous molecular sieve materials and their use in catalysis. Chem. Rev. 1997. Vol. 97, P. 2373.

5. Козлова С.А., Парфёнов В.А., Кирик С.Д. Сорбционные свойства мезопористых силикатов, функционализированных тиольными группами. Журнал СФУ. Техника и технологии. 2015. T. 8(4), C. 494-506. [Kozlova S.A., Parfenov V.A. and Kirik S.D. Adsorption Properties of ThiolFunctionalized Mesoporous Silica. J. Sib. Fed. Univ. Eng. \& Technol. 2015. Vol. 4(8), P. 494-506 (In Russ.)].

6. Taguchi A. and Schuth F. Ordered mesoporous materials in catalysis. Micropor. Mesopor. Mater. 2005. Vol. 77, P. 1-45.

7. De Vos D.E., Dams M., Sels B.F. et al. Ordered mesoporous and microporous molecular sieves functionalized with transition metal complexes as catalysts for selective organic transformations. Chem. Rev. 2002. Vol. 102, P. 3615-3640.

$$
-527-
$$


8. Singh S., Kumar R., Setiabudi H.D. Advanced synthesis strategies of mesoporous SBA-15 supported catalysts for catalytic reforming applications: A state-of-the-art review. Applied Catalysis A, General 2018. Vol. 559, P. 57-74.

9. Soltani R., Dinari M., Mohammadnezhad G. Ultrasonic-assisted synthesis of novel nanocomposite of poly(vinyl alcohol) and amino-modified MCM-41: A green adsorbent for Cd(II) removal. Ultrasonics - Sonochemistry 2018. Vol. 40, P. 533-542.

10. Wan Y. and Zhao D.Y. On the Controllable Soft-Templating Approach to Mesoporous Silicates. Chem. Rev. 2007. Vol. 107, P. 2821-2860.

11. Shen Y., Jiang N., Liu S. et al. Thiol functionalization of short channel SBA-15 through a safe, mild and facile method and application for the removal of mercury (II). J. Environ. Chem. Eng. 2018. Vol. 6, P. 5420-5433.

12. Tenorio M.J., Morère J., Carnerero C. et al. Thiol group functionalization of mesoporous SiO2 SBA-15 using supercritical $\mathrm{CO}_{2}$. Micropor. Mesopor. Mater. 2018. Vol. 256, P. 147154.

13. Hatton B., Landskron K., Whitnall W. et al. Past, present and future of periodic mesoporous organosilicas. Acc. Chem. Res. 2005. Vol. 38, P. 305-312.

14. Appaturi J.N., Johan M.R., Ramalingam R.J. et al. Highly efficient green mesostructured urea functionalized on SBA-15 catalysts for selective synthesis of benzlidenemalononitrile. Micropor. Mesopor. Mater. 2018. Vol. 256, P. 67-74.

15. Wan Y., Zhang D.Q., Zhai Y.P. et al. Periodic mesoporous organosilicas: a type of hybrid support for water-mediated reactions. Chem.-Asian J. 2007. Vol. 2, P. 875-881.

16. Chu H.Q., Yu C., Wan Y. and Zhao D.Y. Synthesis of ordered mesoporous bifunctional $\mathrm{TiO}_{2}-$ $\mathrm{SiO}_{2}$-polymer nanocomposites. J. Mater. Chem. 2009. Vol. 19, P. 8610-8618.

17. Asefa T., MacLachlan M.J., Coombs N. et al. Periodic Mesoporous Organosilicas with Organic Groups Inside the Channel Walls. Nature 1999. Vol. 402, P. 867-871.

18. Sanchez C. and Lebeau B. Design and properties of hybrid organic-inorganic nanocomposites for photonics. MRS Bull. 2001. Vol. 5, P. 377-387.

19. Brown J., Mercier L. and Pinnavaia T.J. Selective adsorption of $\mathrm{Hg}^{2+}$ by thiol-functionalized nanoporous silica. Chem. Commun. 1999, P. 69-70.

20. Liu A.M., Hidajat K., Kawi S. and Zhao D.Y. A new class of hybrid mesoporous materials with functionalized organic monolayers for selective adsorption of heavy metal ions. Chem. Commun. 2000, P. 1145-1146.

21. Zhang L.X., Zhang W.H., Shi J.L. et al. A new thioether functionalized organic-inorganic mesoporous composite as a highly selective and capacious $\mathrm{Hg}^{2+}$ adsorbent. Chem. Commun. 2003, P. 210-211.

22. Nooney R.I., Kalyanaraman M., Kennedy G. et al. Heavy Metal Remediation Using Functionalized Mesoporous Silicas with Controlled Macrostructure. Langmuir 2001. Vol. 17, P. 528-533.

23. Da'na E. Adsorption of heavy metals on functionalized-mesoporous silica: A review. Micropor. Mesopor. Mater. 2017. Vol. 247, P. 145-157.

24. Fellenz N., Perez-Alonso F.J., Martin P.P. et al. Chromium (VI) removal from water by means of adsorption-reduction at the surface of amino-functionalized MCM-41 sorbents. Micropor. Mesopor. Mater. 2017. Vol. 239, P. 138-146. 
25. Козлова С.А. Синтез и исследование селективных сорбентов на основе мезопористых мезоструктурированных силикатов MCM-41 и SBA-15: дис. канд. хим. наук. ИХХT CO РАН, Красноярск, 2013 [Kozlova S.A. The synthesis and investigation of selective sorbents based on mesoporous mesostructured silicates MCM-41 and SBA-15: PhD dissertation. ICCT SB RAS, Krasnoyarsk, 2013 (In Russ.)].

26. Aguado J., Arsuaga J.M. and Arencibia A. Adsorption of aqueous mercury(II) on propylthiolfunctionalized mesoporous silica obtained by cocondensation. Ind. Eng. Chem. Res. 2005. Vol. 44, P. 3665-3671.

27. Choi M., Kleitz F., Liu D.N. et al. Controlled Polymerization in Mesoporous Silica toward the Design of Organic-Inorganic Composite Nanoporous Materials. J. Am. Chem. Soc. 2005. Vol. 127, P. 1924-1932.

28. Lu Y.F., Yang Y., Sellinger A. Self-assembly of mesoscopically ordered chromatic polydiacetylene/silica nanocomposites. Nature 2001. Vol. 410, P. 913-917.

29. Ikegame M., Tajima K. and Aida T. Template Synthesis of Polypyrrole Nanofibers Insulated within One-Dimensional Silicate Channels: Hexagonal versus Lamellar for Recombination of Polarons into Bipolarons. Angew. Chem., Int. Ed. 2003. Vol. 42, P. 2154-2157.

30. Garnweitner G., Smarsly B., Assink R. et al. Self-assembly of an environmentally responsive polymer/silica nanocomposite. J. Am. Chem. Soc. 2003. Vol. 125, P. 5626-5627.

31. Li G.T., Bhosale S., Wang T.Y. et al. Gram-scale synthesis of submicrometer-long polythiophene wires in mesoporous silica matrices. Angew. Chem., Int. Ed. 2003. Vol. 42, P. 3818-3821.

32. Melde B.J., Holland B.T., Blanford C.F. et al. Mesoporous Sieves with Unified Hybrid Inorganic/Organic Frameworks. Chem. Mater. 1999. Vol. 11, P. 3302-3308.

33. Inagaki S., Guan S., Fukushima Y. et.al. Novel Mesoporous Materials with a Uniform Distribution of Organic Groups and Inorganic Oxide in Their Frameworks. J. Am. Chem. Soc. 1999. Vol. 121, P. 9611-9614.

34. Olkhovyk O. and Jaroniec M. Periodic Mesoporous Organosilica with Large Heterocyclic Bridging Groups. J. Am. Chem. Soc. 2005. Vol. 127, P. 60-61.

35. Burleigh M.C., Markowitz M.A., Spector M.S. et al. Amine-Functionalized Periodic Mesoporous Organosilicas. Chem. Mater. 2001. Vol. 13, P. 4760-4766.

36. Landskron K., Hatton B.D., Perovic D.D. et al. Periodic mesoporous organosilicas containing interconnected $\left[\mathrm{Si}\left(\mathrm{CH}_{2}\right)\right]_{3}$ rings. Science 2003. Vol. 302, P. 266-269.

37. Sayari A. and Wang W.H. Molecularly Ordered Nanoporous Organosilicates Prepared with and without Surfactants. J. Am. Chem. Soc. 2005. Vol. 127, P. 12194-12195.

38. Hunks W.J. and Ozin G.A. Periodic Mesoporous Organosilicas with Phenylene Bridging Groups, 1,4- $\left(\mathrm{CH}_{2}\right)_{\mathrm{n}} \mathrm{C}_{6} \mathrm{H}_{4}(\mathrm{n}=0-2)$. Chem. Mater. 2004. Vol. 16, P. 5465-5472.

39. Liu R.L., Shi Y.F., Wan Y. et al. Triconstituent Co-assembly to Ordered Mesostructured Polymer-Silica and Carbon-Silica Nanocomposites and Large-Pore Mesoporous Carbons with High Surface Areas. J. Am. Chem. Soc. 2006. Vol. 128, P. 11652-11662.

40. Hu Q.Y., Kou R., Pang J.B. et al. Mesoporous carbon/silica nanocomposite through multicomponent assembly. Chem. Commun. 2007, P. 601-603.

41. Aguado J., Arsuaga J.M., Arencibia A. Influence of synthesis conditions on mercury adsorption capacity of propylthiolfunctionalized SBA-15 obtained by co-condensation. Micropor. Mesopor. Mater. 2008. Vol. 109, P. 513-524. 
42. Hoang V.D., Dang T.P., Dinh Q.K. et al. The synthesis of novel hybrid thiol-functionalized nano-structured SBA-15. Adv. Nat. Sci.: Nanosci. Nanotechnol. 2010. Vol. 1(3), P. 035011.

43. Arencibia A., Aguado J., Arsuaga J.M. Regeneration of thiol-functionalized mesostructured silica adsorbents of mercury. Appl. Surf. Sci. 2010. Vol. 256, P. 5453-5457. 